\section{A topoisomerase ll-dependent G2 cycle checkpoint in mammalian cells}

C. Stephen Downes, Duncan J. Clarke, Ann M. Mullinger, Juan F. Giménez-Abián, Andrew M. Creighton \& Robert T. Johnson

Nature 372, 467-470 (1994)

DURING production a stray line was introduced across panel $c$ of Fig. 4 of this Letter. The figure is reproduced below.
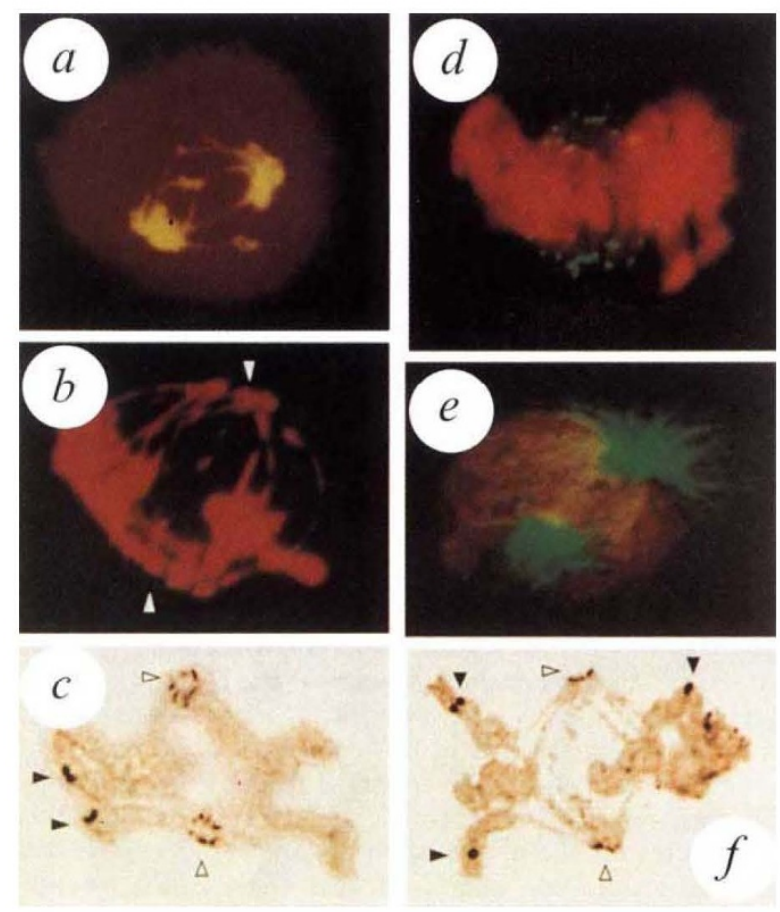

\section{GUIDE TO AUTHORS}

PLEASE follow these guidelines so that your manuscript may be handled expeditiously.

Nature is an international journal covering all the sciences. Contributors should therefore bear in mind those readers who work in other fields and those for whom English is a second language, and write clearly and simply, avoiding unnecessary technical terminology. Space in the journal is limited. making competition for publication severe. Brevity is highly valued. One printed page of Nature, without display items, contains about 1,300 words.

Manuscripts are selected for publication according to editorial assessment of their suitability and reports from independent referees. They can be sent to London or Washington and should be addressed to the Editor. Manuscripts may be dealt with in either office, depending on the subject matter and will whe matter, and will where necosary be sent between offes briority canno be given to pres one-page fax. All manuscripts are acknowledged on recipt but fewer than half are sent for review. Thos that are not reviewed are returned as rapidly as possible so that they may be submitted elsewhere without delay. Contributors may suggest reviewers; limited requests for the exclusion of specific reviewers are usually heeded. Manuscripts are usually sent to two or three reviewers, chosen for their expertise rather than their geographical location. Manuscripts accepted for publication are processed from the London office.

Nature requests authors to deposit sequence and crystallographic data in the databases that exist for this purpose.

Once a manuscript is accepted for publication, contributors will receive proofs in about 4 weeks. Nature's staff will edit manuscripts with a view to brevity and clarity, so contributors should check proofs carefully. Manuscripts are generally published 23 weeks after receipt of corrected proofs. Nature does not exact page charges. Contributors receive a reprint order form with their proofs; reprint orders are processed after the manuscript is published and payment received.

\section{Categories of paper}

Review Articles survey recent developments in a field. Most are commissioned but suggestions are welcome in the form of a one-page synopsis addressed to the Reviews Coordinator. Length is negotiable in advance.

Progress articles review particularly topical developments for a nonspecialist readership. They do not exceed 4 pages in length. Suggestions may be made to the Reviews Coordinator in the form of a brief synopsis.

Articles are research reports whose conclusions are of general interest and which are sufficiently rounded to be a substantial advance in understanding. They should not have more than 3,000 words of text (not including figure legends) or more than six display items and should not occupy more than five pages of Narure.

Articles start with a heading of $50-80$ words written to advertise their content in general terms. to which editors will pay particular attention. The heading does not usually contain numbers. abbreviations or measurements. The introduction to the study is contained in the first two or three paragraphs of the article, which also briefly summarize its results and implications. Articles have fewer than 50 references and may contain a few short subheadings.

Letters to Nature are short reports of outstanding novel findings whose implications are general and important enough to be of interest to those outside the field. Letters should have 1,000 or fewer words of text and four or fewer display items. The first paragraph describes. in not more than 150 words and without the use of abbreviations, the background, rationale and chief conclusions of the study for the particular benefit of non-specialist readers. Letters do not have subheadings and contain fewer than 30 references.

Commentary articles deal with issues in, or arising from, research that are also of interest to readers outside research.

Nows and Vlews articles inform nonspecialist readers about new scientific advances, sometimes in the form of a conference report. Most are commissioned but proposals can be made in advance to the News and Views Editor.

Scientific Correspondence is for discussion of topical scientific matters, including those published in Nature, and for miscellaneous contributions. Priority is given to letters of fewer than 500 words.

\section{Preparation of manuscripts}

All manuscripts should be typed. double-spaced, on one side of the paper only. An original and four copies are required, each accompanied by artwork. If photographs are included, five sets of originals are required; for line drawings, one set of originals and four good-quality photocopies are acceptable. Reference lists, figure legends and tables should all be on separate sheets, all of which should be double-spaced and numbered. Three copies of relevant manuscripts in press or submitted for publication elsewhere should be included with submitted manuscripts, clearly marked as such Five copies of revised and resubmitted manuscripts, labelled with their manuscript numbers, are required, together with five copies of a letter detailing the changes made.

Titles are brief and simple. Active verbs, numerical values, abbreviations and punctuation are to be avoided. Titles should contain one or two key words for indexing purposes.

avoided. Thork should be marked individually and clearly with the author's name and, when known, the manuscript number. Ideally, no figure should be larger than 28 by $22 \mathrm{~cm}$. Figures with several parts manuscript number. Ideally, no figure should be larger than 28 by $22 \mathrm{~cm}$. Figures with several parts
are to be avoided and are permitted only if the parts are closely related, either experimentally or are to be avoided and are permitted only if the parts are closely related, either experimentally or
logically. Unlettered originals of photographs should be provided. Suggestions for cover illustrations. with captions and labelled with the manuscript number, are welcome. Original artwork is returned when a manuscript cannot be published.

Protein/nucleotide sequences should ideally be in the three-letter and not the single-letter code for amino acids. One column width of Nature can accommodate 20 amino acids or 60 base pairs. Colour artwork. A charge of $£ 500$ per page is made as a contribution towards the cost of reproducing colour figures. Inability to pay these costs will not prevent publication of essential colour figures if the circumstances are explained. Proofs of colour artwork may be sent to contributors under separate cover from their galley proofs.

Figure legends should not exceed 300 words and ideally should be shorter. The figure is described Figure legends should not exceed 300 words and ideally should be shorter. The figure is described
first, then, briefly, the method. Reference to a method published elsewhere is preferable to a full description. Methods are not described in the text.

References are numbered sequentially as they appear in the text, followed by those in tables and finally by those in figure legends. Only papers published or in the press are numbered and included in the reference list. All other forms of reference should be cited in the text as a personal communication. manuscript submitted or in preparation. Text is not included in reference lists. References are abbreviated according to the World List of Scientific Periodicals (Butterworths, London. 1963-65). The first and last page numbers are included: reference to books includes publisher, place and date. Abbreviations, symbols, units and Greek letters should be identified the first time they are used. Acronyms should be avoided whenever possible and, if used, defined. Footnotes are not used in the Acrony

Acknowledgements are brief and appear after the reference list; grant and contribution numbers ack not allowed.

Supplementary information is material relevant to Articles or Letters which cannot, for lack of space, be published in full, but which is available from Nature on request

Submission. Manuscripts can be sent to the Editor at 4 Little Fssex Street, London WC2R 3LF. UK or at 1234 National Press Building. Washington, DC 20045, USA. A telephone and fax number should be included. Manuscripts or proofs sent by air courier to London should be declared as 'manuscripts' and 'value $\$ 5$ ' to prevent the imposition of import duty and value-added tax.
THE contents list in this (the 10 November) issue included a typographical error. 